gr-qc/9705065

McGill/97-09

\title{
Pure states don't wear black
}

\author{
Robert Myers ${ }^{1}$ \\ Department of Physics \\ McGill University \\ Montréal, Québec H3A-2T8 Canada
}

\begin{abstract}
Recently, string theory has provided some remarkable new insights into the microphysics of black holes. I argue that a simple and important lesson is also provided with regards to the information loss paradox, namely, pure quantum states do not form black holes! Thus it seems black hole formation, as well as evaporation, must be understood within the framework of quantum decoherence.
\end{abstract}

1 Internet: rcm@hep.physics.mcgill.ca 
Over twenty years ago, Stephen Hawking[1] made the remarkable discovery that quantum mechanics leads to the emission of thermal radiation from classical black holes. A vexing puzzle concerning the compatibility of quantum theory and general relativity was raised shortly afterwards by the question: What is the final end state which is produced when a pure quantum state collapses to form a black hole which then emits thermal radiation? Hawking[2] proposed that the black hole continues radiating until, having radiated away its entire mass, it disappears, leaving behind only thermal radiation in a mixed quantum state. In this progression from a pure to a mixed state, the details of the original state are lost and unitary time evolution, a basic tenet of quantum theory, is violated. Hence this proposal suggests that the basic principles of quantum physics, while successful in describing everyday experiments, cannot accomodate gravity without radical revision. Attempts to produce a less distressing scenario in which information of the original state is preserved have proven unsatisfactory[3], and so this disturbing puzzle, the information paradox, remains unsolved.

A related puzzle arises because combining Hawking's result with the classical laws of black hole mechanics [4] indicates that, as anticipated by Bekenstein [5], black holes have an intrinsic entropy proportional to the surface area of their horizons [6]:

$$
S_{B H}=A_{H} / 4 G
$$

In this context, one has a thermodynamic understanding of this entropy, i.e., it is related to energy unavailable for work. A longstanding problem has been finding a statistical mechanical understanding of this entropy in terms of some microscopic degrees of freedom. Recently, superstring theory has provided some extraordinary new insights into this question.

This progress ensued from the realization that in string theory extended objects other than strings also play an important role. In particular, Dirichlet branes, or D-branes [7], form a diverse class of extended objects, distinguished by a simple description in the framework of perturbative or weakly-interacting strings. Yet D-branes exhibit a rich dynamics, 
including a wide variety of complicated bound states. Characterizing these bound states by certain asymptotic features, e.g., mass and charges, the degeneracy $\mathcal{D}$ of these configurations is amenable to statistical mechanical analysis within the perturbative regime. If the string coupling constant (and hence also Newton's constant for the theory) is increased, it is believed that the bound states undergo gravitational collapse to form black holes. In this complementary strong-coupling regime, one can use the low-energy string equations — essentially Einstein gravity coupled to various massless fields - to determine the corresponding black hole solution, for which one can calculate the Bekenstein-Hawking entropy (11). Given appropriate arguments (e.g., supersymmetry) that the degeneracy is invariant when the coupling changes, one might expect $S_{B H}=\ln \mathcal{D}$. These calculations were first elucidated for a class of extremally charged black holes in five dimensions[8], and yielded a striking agreement between $S_{B H}$ and the statistical entropy. This analysis was quickly extended to a variety of other configurations, including black holes in four dimensions [9] and slightly non-extremal black holes [10]. Again a precise agreement of the entropies was found in each case.

Suprisingly, it was further found that the D-branes described the dynamics of nearextremal black holes, at least at low energies [11]. In particular, it was shown that the black hole decay rates and absorption cross-sections were precisely reproduced. As a simple example, the absorption cross-section for a scalar (with energy $\omega$ ) incident on an extremal D-brane configuration is

$$
\sigma_{\mathrm{abs}}=A_{H} \beta \omega\left(\left\langle n_{\omega / 2}\right\rangle+1\right)
$$

where $A_{H}$ is the quantity that matches to the horizon area, while $\beta$ is a scale which characterises the density of states of the D-brane configurations. Finally, $n_{\omega / 2}$ is the number of internal excitations with energy $\omega / 2$ in a given configuration. To good accuracy, $\left\langle n_{\omega / 2}\right\rangle$ averaged over all of the degenerate D-brane configurations is approximated by an 
effective canonical ensemble

$$
\left\langle n_{\omega / 2}\right\rangle=\frac{1}{e^{\beta \omega}-1} .
$$

This result precisely reproduces the cross-section for the corresponding extremal black hole[11]. In particular for small frequencies $(\beta \omega<<1)$, one has the finite result $\sigma_{\mathrm{abs}}=A_{H}$, a universal result for black holes 12. Thus the D-branes give a very robust model of nearextremal black hole dynamics.

Given that the D-branes provide such a successful model of black hole microphysics, one might ask if there are any lessons to be learned with regard to the information paradox. Here I argue that they provide a simple but important message: pure quantum states do not form black holes!

First, the entropy calculations have shown that we should take seriously the interpretation of $S_{B H}$ as a statistical mechanical entropy. Hence, with a pure state, since the entropy vanishes, one must have that $A_{H}=0$, i.e., there is no horizon and no black hole. In the D-brane calculations, it is only by considering a mixed state including all of the degenerate configurations that the black hole results are matched.

The requirement of a mixed state becomes even more explicit in the scattering calculations. In order to produce the correct black hole correspondence for, e.g., the absorption cross-section (2), one calculates with a decoherent ensemble of all degenerate D-brane configurations. In particular, any generic tampering with the low energy behavior $\left\langle n_{\omega / 2}\right\rangle \simeq 1 / \beta \omega$ would destroy the correspondence to the universal black hole cross-section.

The precise connection between D-brane configurations at weak-coupling and gravitational field solutions at strong-coupling is not well understood. Certain qualitative aspects are clear, e.g., these configurations develop strong gravitational fields over a range much larger than the string scale. The original expectation was that, having prescribed a given set of asymptotic charges, there is a unique black hole solution with those same charges and any of the corresponding D-brane configurations will yield this same black hole.

While the uniqueness of the black hole solution may be true - although it remains to 
be rigorously proven - a given set of charges simply do not fix a unique field configuration. Actually, there are infinite families of solutions, which, however, contain singular null surfaces which are not concealed behind any event horizons [13] [14]. Given that pure (or even generic mixed) states of D-branes do not appear in strong-coupling to correspond to a black hole, it is thus clear that these non-black solutions provide a vast collection of alternatives.

Since the prospect of dealing with naked singularities must seem an unsavory prospect to most relativists, several comments are in order. First, when dealing with a system composed of a single type of D-brane, it is accepted that the corresponding strong-coupling solutions have such singular surfaces. One expects in a certain sense that string theory is telling us about the resolution of these singularities with D-branes. Further, when I say there are "singularities" present, I mean the solutions of the leading-order low-energy string equations are singular. The full string equations will modify the strong curvature regions, and at the "singularity" the full stringy nature of these configurations should become manifest. There it is likely that even the notion of a spacetime metric must be discarded.

I would add that further interesting evidence in support of these arguments can be found in [13]. There, in an ensemble of D-brane configurations, the contributions are weighted in order to consider a nonuniform charge density. Hence the degeneracy is reduced, but this is precisely matched by a reduction of the horizon area, maintaining the expected equivalence $S_{B H}=\ln \mathcal{D}$. However, one finds that even this benign tampering with the mixed state produces a mild curvature singularity at the horizon 15.

Given that pure states do not form black holes, it may seem that only exceptional circumstances can lead to black hole formation within a quantum framework. This is, of course, not the case. Rather only the standard mechanisms of decoherence are required [16]. As a practical matter, if some segment of a quantum system is considered, a pure initial state can appear to become a mixed state through random interactions with its environ- 
ment. For example, in the D-brane model, if a given observer is unable or chooses not to observe low energy radiation below some energy scale $\omega$, one can estimate from (2) that a mixed state forms on a decoherence time-scale $T^{-1}>\omega^{7}\left(A_{H} \beta\right)^{2}$ (in four-dimensional spacetime). For a fixed $\omega$, the decoherence time-scale becomes especially short at strongcoupling. Thus, as a practical matter, pure D-brane states rapidly evolve to mixed states, which would then display the characteristics of a black hole. There must be strong correlations, however, between the "black hole state" and its environment even at its inception.

To summarize, then, recent progress in understanding black hole microphysics carries a simple message: pure quantum states do not form black holes. Rather, such states are likely to correspond to field configurations with "singularities", strong curvature regions, which are not clothed by horizons. Thus the information paradox results from an illposed question. In completely understanding black hole evaporation, one is still likely to face information loss questions. However, the discussion must now be formulated in a framework where black holes are inherently associated with mixed states. In this approach, the resolution of these questions seems likely not to require new physics incorporating information loss as a matter of principle. Rather, it seems likely that the information loss arises as a common matter of practice, with which we are familiar in our everyday lives.

I thank James Anglin and Ted Jacobson for useful comments, and Ramzi Khuri for suggestions on improving the manuscript. I would also like to thank Peter Haagensen for his efforts in submitting my essay on time. This research was supported in part by NSERC of Canada and Fonds FCAR du Québec. 


\section{References}

[1] S.W. Hawking, Commun. Math. Phys. 43 (1975) 199.

[2] S.W. Hawking, Phys. Rev. D14 (1976) 2460.

[3] see for example: J. Preskill, "Do black holes destroy information," preprint hepth/9209058.

[4] J.M. Bardeen, B. Carter and S.W. Hawking, Commun. Math. Phys. 31 (1973) 161.

[5] J.D. Bekenstein, Phys. Rev. D7 (1973) 2333; D9 (1974) 3292.

[6] This result also applies for Einstein gravity in higher spacetime dimensions, in which case the surface area $A_{H}$ refers to the volume of a space-like cross-section of the horizon, e.g., in five dimensions, $A_{H}$ becomes a three-dimensional volume.

[7] J. Polchinski, "TASI Lectures on D-branes," e-print hep-th/9611050; J. Polchinski, S. Chaudhuri and C.V. Johnson, "Notes on D-branes," e-print hep-th/9602052.

[8] A. Strominger and C. Vafa, Physics Letters B379 (1996) 99 hep-th/9601029.

[9] C.V. Johnson, R.R. Khuri and R.C. Myers, Phys. Lett. B378 (1996) 78 hepth/9603061]; J.M. Maldacena and A. Strominger, Phys. Rev. Lett. 77 (1996) 428 hep-th/9603060.

[10] C.G. Callan and J.M. Maldacena, Nucl. Phys. B472 (1996) 591 hep-th/9602043]; G.T. Horowitz and A. Strominger, Phys. Rev. Lett. 77 (1996) 2368 [hep-th/9602051].

[11] S.R. Das and S.D. Mathur, Nucl. Phys. B478 (1996) 561 hep-th/9606185; Nucl. Phys. B482 (1996) 153 [hep-th/9607149]; J. Maldacena and A. Strominger, Phys. Rev. D55 (1997) 861 hep-th/9609026.

[12] S.R. Das, G.W. Gibbons and S.D. Mathur, Phys. Rev. Lett. 78 (1997) 417 hepth/9609052].

[13] G.T. Horowitz and D. Marolf, Phys. Rev. D55 (1997) 846 hep-th/9606113; Phys. Rev. D55 (1997) 835 hep-th/9605224.

[14] N. Kaloper, R.C. Myers and H. Roussel, "Wavy Strings: Black or Bright?", to appear in Physical Review D, e-Print hep-th/9612248; A.A. Tseytlin, Mod. Phys. Lett. A11 (1996) 689 hep-th/9601177.

[15] G.T. Horowitz and H.-S. Yang, "Black Strings and Classical Hair," e-Print hepth/9701077.

[16] W.H. Zurek, Physics Today 44, No. 10 (1991) 36. 